\title{
Evaluation de la productivité des herbacées fourragères des forêts sacrées de Koupéla dans le Centre Ouest du Burkina Faso
}

\author{
Yahaya SAMANDOULGOU ${ }^{1,3^{*}}$, Halidou COMPAORE ${ }^{1}$, Sibiri Jean ZOUNDI $^{1}$ et \\ Yvette Chantal ZOUNGRANA-KABORE ${ }^{2,3}$ \\ ${ }^{1}$ Institut de l'Environnement et de Recherches Agricoles (INERA), 04 BP 8645 Ouagadougou 04, \\ Burkina Faso. \\ ${ }^{2}$ Université Polytechnique de Bobo-Dioulasso, Burkina Faso. \\ ${ }^{3}$ Laboratoire d'Etude et de Recherche des Ressources Naturelles et des Sciences de l'Environnement \\ (LERNSE/UPB) ; Direction de l'Agence Nationale de Biosécurité du Burkina Faso. \\ *Auteur correspondant; E-mail: samandoulgou@yahoo.fr; Tel : (+226) 70723248
}

\section{RESUME}

Les forêts sacrées du village de Koupéla, situées dans la région du Centre Ouest du Burkina Faso, sont pâturées par le cheptel herbivore domestique. La présente étude évalue la productivité des herbacées fourragères des forêts sacrées. Deux sites expérimentaux situés dans une dépression et sur un glacis sont comparés à un autre sur jachère comme témoin. Conduite selon la méthode des points quadrats, l'étude a permis d'effectuer dans les parcours (i) un relevé floristique de la végétation herbacée, (ii) une évaluation de la phytomasse épigée. Il a été identifié par cet inventaire floristique 101 espèces sur les 3 sites de relevés. Les Poacées ont eu une meilleure contribution spécifique (CS) dans toutes les unités de pâturage. Spécifiquement, la zone sacrée compte un grand nombre d'herbacées pérennes dont Andropogon gayanus, Andropogon ascinodis. Cymbopogon giganteus et Cymbopogon schoenanthus. La biomasse nette épigée des herbacées est évaluée à 4029,76 kg/ha en moyenne pour les forêts sacrées contre 2195,25 kg/ha pour la jachère. La capacité de charge en saison pluvieuse est estimée à 2,50 UBT/ha/ pour la dépression contre 1,17 UBT/ha pour la jachère. La présente étude suggère des évaluations complémentaires du potentiel fourrager global du terroir afin de proposer une meilleure formule de gestion des ressources fourragères de ces forêts sacrées.

(C) 2019 International Formulae Group. All rights reserved.

Mots clés : Forêts sacrées, herbacées fourragères, cheptel, jachère, valeur pastorale, Koupéla, Burkina Faso.

\section{Evaluation of herbaceous forage productivity of the Koupéla sacred forests in West-Central Burkina Faso}

\begin{abstract}
Sacred forests of the village of Koupela, an ecological ensemble located in the Central West region of Burkina Faso, are grazed by the domestic herbivorous livestock of this village. This study assesses the productivity of herbaceous forage in sacred forests. Two experimental sites located in a depression and a glacis are compared to another on fallow as a control. Conducted according to the quadratic points method, the study made it possible to carry out in the courses (i) a floristic survey of the herbaceous vegetation, (ii) an evaluation
\end{abstract}


of the epiged phytomass. It has been identified through this floristic inventory 101 species on the 3 survey sites. Globally Poaceae had better specific contributions (CS) in all grazing units. Specifically, the sacred area has a large number of perennial grasses including Andropogon gayanus, Andropogon ascinodis, Cymbopogon giganteus. and Cymbopogon schoenanthus. The net above-ground biomass of herbaceous species is estimated at $4029.76 \mathrm{~kg}$ / ha on average for sacred forests against $2195.25 \mathrm{~kg} /$ ha for fallow. The carrying capacity in the rainy season is estimated at $2.50 \mathrm{UBT} / \mathrm{ha} /$ for the depression against $1.17 \mathrm{UBT} /$ ha for the fallow. This study suggests complementary assessments of the global forage potential of the terroir in order to propose a better formula for managing the forage resources of these sacred forests.

(C) 2019 International Formulae Group. All rights reserved.

Keywords: Sacred forests, herbaceous forages, livestock, fallow, pastoral value, Koupéla, Burkina Faso.

\section{INTRODUCTION}

Soumis aux effets des changements climatiques, le Burkina Faso à l'instar des autres pays sahéliens, connait de fortes dégradations des ressources naturelles et plus particulièrement des terres. En plus des facteurs climatiques, les terres agricoles subissent les effets de la pression démographique avec comme corollaire une gestion concurrentielle voire conflictuelle. Ce phénomène est particulièrement vécu dans le terroir de Koupéla, notre site d'étude, où les bas-fonds et les terres de dépression, jadis réservés à la pâture, sont de jour en jour envahis par les cultures (Kabirou, 2016; Koutchika et al., 2013). Les forêts sacrées sont des sites longtemps considérés comme des lieux de culte et d'initiation, d'espaces de méditations, de prises de décisions notables, de cimetières des ancêtres et lieux de recueillement (Esoh, 2003). Ainsi, le caractère sacré ou culturel porté par les populations locales à ces aires de conservation communautaire représente un avantage pour la conservation durable, mais aussi un handicap à la valorisation et à la gestion durable de ces lieux (Kabirou, 2016). En fait, au Burkina Faso, sur le plan institutionnel (Code de l'environnement, Réforme agraire, Plan d'Investissement Forestier, Code forestier, etc.) très peu d'égards a été porté aux îlots forestiers sacrés en comparaison aux forêts classées considérées comme un patrimoine de l'Etat. Les forêts sacrées ont été décrites dans de nombreux travaux en Afrique (César et al., 2011 ; CAPES/RGCB, 2006 ; Esoh, 2006), mais les résultats ont rarement porté sur leur utilisation par les éleveurs. De nos jours, en raison du surpâturage et de la dégradation des ressources naturelles liés aux facteurs anthropiques et aux effets du changement climatique, les îlots forestiers sacrés constituent une zone de pâture pour le bétail. Une meilleure connaissance de la distribution et de la structure, de la diversité et de la dynamique herbacées fourragères dans ces forêts pourraient inciter la population et les autorités à mettre en place un système d'aménagement et de valorisation.

Kokou et al. (2005) ont montré la fragilité actuelle des systèmes de gestion locale des forêts sacrées que les populations avaient elles-mêmes protégées depuis plusieurs générations. Cela a été favorisé par le développement des religions monothéistes importées et l'installation d'immigrants (Lasseur, 2010). Les éleveurs profitent de la faiblesse du système social de gestion des forêts sacrées pour y faire pâturer leurs cheptels. Ce sont en général des migrants venus du Nord du pays pour s'installer dans le village ou des transhumants à la recherche de fourrage pour leurs animaux (Ouedraogo et al., 2010). L'hypothèse de notre étude présage que les forêts sacrées de Koupéla présentent un pâturage plus fourni en fourrages herbacés en saison des pluies que les autres parcours notamment les jachères. C'est pourquoi, ces sites indigènes de conservation et de préservation de la diversité biologique sont constamment pâturés. Très peu d'études se sont intéressées à cette fonction nouvelle des forêts sacrées qui est celle de l'alimentation des animaux domestiques. La présente étude vise à évaluer dans un premier temps la qualité de la végétation herbacée des forêts sacrées puis sa production par unité de surface 
et enfin établir la capacité de charge de ces sites comparés aux jachères.

\section{MATERIEL ET METHODES \\ Site d'étude}

Le village de Koupéla, situé entre $11^{\circ}$ $58^{\prime} 0^{\prime \prime}$ Nord et $2^{\circ} 23^{\prime} 0^{\prime \prime}$ Ouest (Figure 1), a une population de 1507 habitants, répartie entre trois ethnies, les Gourounsis, les Mossi et les Peuls. Selon MED (2007), la densité de la population de Koupéla est, à l'image du Plateau central du Burkina Faso, 80,9 habitants au $\mathrm{km}^{2}$, supérieure à la moyenne nationale $\left(51,8\right.$ habitants au $\left.\mathrm{km}^{2}\right)$. Selon Kabirou (2016) le village compte 5 forêts sacrées contigües situées autour du village classées «forêts sacrées des ancêtres » selon la typologie de Kokou et Sokpon (2006). Elles couvrent une superficie totale de 28,4 ha composé de 10,4 ha de glacis et 18 ha dans une dépression ripicole (Coulibaly, 2016). Une partie de ces forêts sacrées est située dans une dépression traversée par une rivière tarissant entre avril et mi-mai. Cette disponibilité en eau en plus de celle du pâturage, attirent un grand nombre d'animaux $\mathrm{du}$ village et des autres terroirs surtout en saison sèche (Compaore, 2018).

Selon la subdivision phytogéographique du Burkina Faso faite par Fontes et Guinko (1995), la végétation de la province du Boulkiemdé, dont relève le village de Koupéla, est de type nord soudanien caractérisé par des savanes à graminées annuelles, à arbres et arbustes.

\section{Méthodes utilisées et paramètres mesurés Relevés de la végétation}

L'évaluation de la dynamique de la végétation a été faite dans la forêt sacrée sur deux sites différents par leur physionomie en comparaison à une situation témoin représentée par une ancienne jachère. Les deux sites expérimentaux comprennent une dépression et un glacis. Dans chacun des trois sites (deux expérimentaux plus le témoin), une parcelle écologique d'observation couvrant une superficie d'un hectare (Toutain et De Wispelaere, 1978 ; Toutain et Piot, 1980) a été délimitée.

Des inventaires de la végétation herbacée ont été réalisés à l'aide de la méthode des «points quadrats alignés » (Daget et Poissonet, 1971) suivant un réseau de lignes de $20 \mathrm{~m}$ matérialisées par des piquets métalliques. L'évaluation floristique a été effectuée sur la ligne de cordon dans le sillon de sous solage, au centre des sillons. Sur chaque point de suivi, 100 relevés ont été effectués. Le nombre de lignes a été limité à 20 à chaque fois, étant donné qu'une précision satisfaisante est atteinte avec cette limite (Daget et Poissonet, 1971).

Une fiche d'enregistrement des données a servi à noter les noms des espèces annoncées (en contact avec la tige métallique). Chaque colonne est consacrée à un point du double décamètre et les espèces annoncées sont cochées par une croix dans la case correspondante. La méthode permet ainsi de calculer : (i) la fréquence spécifique (FS), valeur absolue, correspondant à la proportion des espèces au niveau du sol ; (ii) la contribution spécifique (CSi) de chaque espèce définie comme le rapport de la fréquence spécifique (FSi) de cette espèce à la somme des FSi de toutes les espèces recensées sur 100 points échantillonnés.

$$
C S i=\frac{F S i}{\sum_{i=1}^{n} F S} \times 100
$$

Avec $\mathrm{n}=$ nombre d'espèces. CSi est la fréquence relative de l'espèce i dans l'ensemble des fréquences spécifiques observées.

$\mathrm{La}$ détermination $\mathrm{du}$ nombre d'observations est effectuée par le calcul de l'intervalle de confiance à partir de l'effectif cumulé ligne par ligne des contacts de l'espèce dominante sur l'effectif cumulé des contacts enregistrés par l'ensemble des espèces (Daget et Poissonet, 1971).

$$
\text { I } C= \pm 2 \sqrt{\frac{n(N-n)}{N^{2}}}
$$

Avec N l'effectif cumulé des contacts de l'ensemble des espèces et « $\mathrm{n}$ »l'effectif cumulé des contacts de l'espèce dominante

\section{Phytomasse}

Le matériel utilisé pour la récolte de la phytomasse se compose : d'un cadre métallique carré de $1 \mathrm{~m}^{2}$, d'une balance de capacité de $5 \mathrm{~kg}$ avec une précision de $10 \mathrm{~g}$, d'un sécateur et de sacs en tissu pour 
respectivement la fauche et la collecte de végétaux. La phytomasse a été évaluée par la méthode de la récolte intégrale sur des placeaux de $1 \mathrm{~m}^{2}$ à raison de 40 prélèvements dans chaque parcelle en fin octobre (période où la biomasse est maximale).

Les prélèvements ont été effectués de façon aléatoire dans toutes les parcelles. Les poids de la biomasse fraiche des échantillons ont été mesurés immédiatement après la fauche. Par la suite, la teneur en eau est déterminée sur plusieurs échantillons par dessiccation à l'étuve à $85^{\circ} \mathrm{C}$ jusqu'à l'obtention du poids constant (Levang et Grouzis, 1980)

\section{Estimation de la capacité de charge}

Les capacités de charge (CC) ont été calculées sur la base des biomasses consommables (Boudet, 1991).

\section{$C C=(\mathrm{PB} \times \mathrm{U}) /(6,25 \mathrm{xt})$}

avec: $\mathrm{CC}=$ Capacité de Charge ; $\mathrm{PB}=$ production brut (MS $/ \mathrm{kg} / \mathrm{ha}, \quad \mathrm{U}=$ Taux d'utilisation (estimé à $40 \%$, pour la zone sahélienne); $\mathrm{t}=$ temps d'utilisation (jour) 6,25 $=$ Consommation de l'UBT en $\mathrm{Kg}$ de MS/jour ; UBT = Unité Bétail Tropical.

\section{Valeur pastorale}

L'indice de valeur pastorale brute $(\mathrm{VPb})$ des communautés, des parcelles a été calculé à partir des contributions spécifiques de la végétation herbacée et des Indices de qualité Spécifiques de l'espèce i (ISi). Une $\mathrm{VPb}$ est établie sur une échelle de cotation de 0 à 3 (Akpo et al, 2002). Sont qualifiées de plantes à bonne valeur pastorale (bonne $\mathrm{VPb}$ ) celles dont l'ISi est égal à 3 ; de plantes de moyenne valeur pastorale (Moyenne $\mathrm{VPb}$ ), celles dont l'Isi est égal à 2 et de plantes de Faible valeur pastorale (Faible VPb), celles dont l'Isi est égal à 1 . Enfin, les plantes sans valeur pastorale (Sans $\mathrm{VPb}$ ), sont celles dont l'Isi est égal à 0 .

L'indice spécifique traduit l'intérêt zootechnique, c'est-à-dire la valeur bromatologique de chaque espèce végétale. La détermination de la valeur pastorale repose sur l'appétibilité, la productivité (kg MS/ha/jour) et la valeur nutritive des espèces obtenues à partir de la composition chimique. Pour le calcul, la formule utilisée est celle proposée par Daget et Poissonet (1971) :

$$
\mathrm{VPb}=0,2 \Sigma \quad \mathrm{CSi} \mathrm{xIsi} \quad(4)
$$

Avec: Csi : Contribution spécifique, Isi : Indice spécifique de l'espèce i, noté de 0 à 3 ; 0,2 est un coefficient.

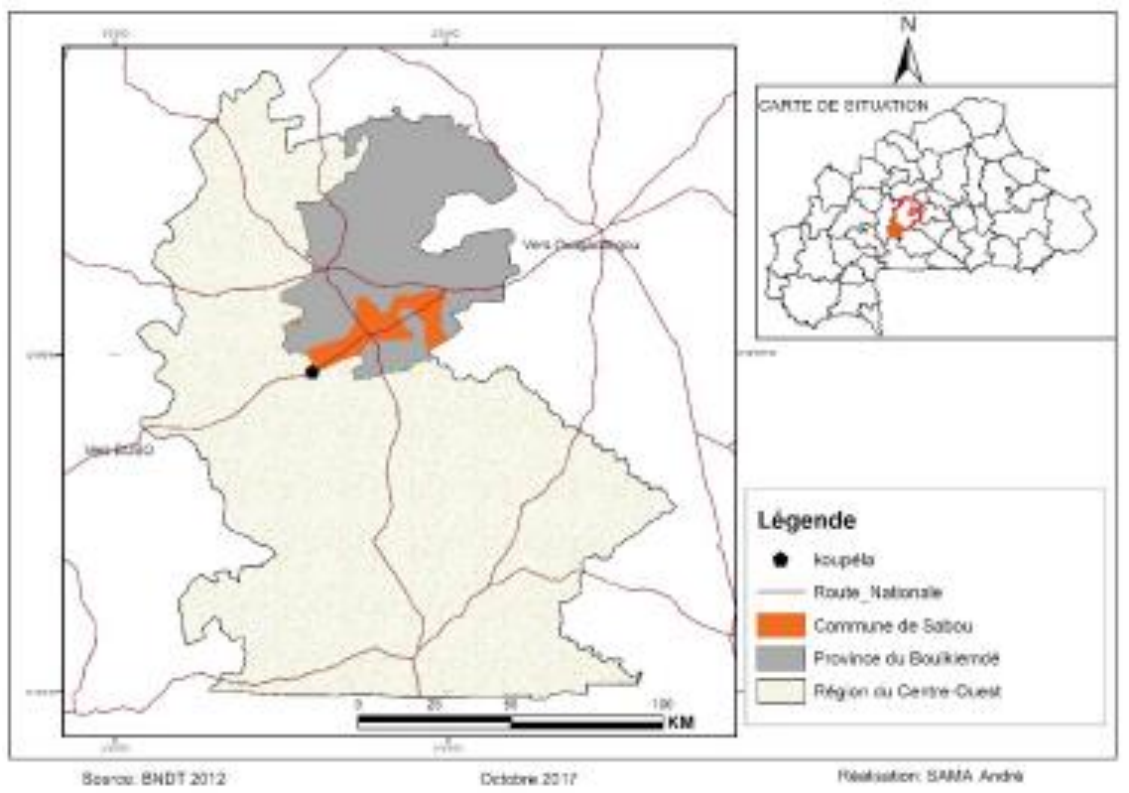

Figure 1 : Localisation de la zone d'étude. 


\section{RESULTATS}

\section{Composition floristique de la végétation herbacée \\ Diversité spécifique}

Le Tableau 1 présente les principales familles sur les différentes unités de production fourragère. La famille des Poaceae est la plus représentée avec 28 espèces dans la dépression, 26 sur le glacis et 7 dans la jachère. La seconde famille importante est constituée des Fabaceae avec 12,8 et 4 espèces respectivement pour la dépression, le glacis et la jachère. Cent une (101) espèces herbacées ont été recensées dans la partie dépression de la forêt sacrée, 87 sur le glacis et 27 sur la jachère. Ainsi, la jachère ne compte qu'un tiers du nombre moyen des espèces relevées dans les forêts sacrées.

\section{Contribution spécifique des différentes catégories de fourrages et des principales espèces \\ Contribution spécifique des catégories de fourrages}

A partir des observations sur le terrain, les herbacées ont été regroupées en grandes familles que sont les Poaceae, les légumineuses, et autres herbacées. Dans les parcours inventoriés, les Poaceae dominent dans tous les sites avec 66,16\% en moyenne dans la forêt sacrée et 33,04\% dans la jachère (Tableau 2). Les espèces classées comme " autres herbacées », ont une contribution spécifique allant de $16,22 \%$ en moyenne pour la forêt sacrée à plus de $50 \%$ dans la jachère. Les légumineuses composent $16,29 \%$ des herbacées de la jachère contre $12,08 \%$ et $7,98 \%$ respectivement pour la dépression et le glacis en forêts sacrées

\section{Contribution spécifique (en \%) des principales espèces}

Du point de vue spécifique, les herbacées des forêts sacrées sont dominées par les graminées annuelles à l'exception de Andropogon gayanus dans les zones de dépression (Tableau 3). L'espèce Andropogon pseudapricus, espèce peu appétée, se démarque dans tous les parcours par une relative bonne contribution spécifiques $14,15 \%, 16,21 \%$ et $17,14 \%$ respectivement pour la zone de dépression, de glacis et de jachère. La zone de dépression et le glacis comportent les meilleures herbacées fourragères que sont Zornia glochidiata, Eragrostis tenella, Andropogon gayanus, Alysicarpus ovalifolius et Rottboellia exaltata. Les jachères sont dominées par espèce non appétée par les animaux Hyptis suaveolens $(28,15 \%)$ Abutilon panosum $(17,9 \%)$

\section{Valeur pastorale (VP)}

Dans la dépression, la valeur pastorale brute calculée est plus élevée $(54,03 \%)$ suivie du glacis $(47,9 \%$ et enfin de la jachère $(34,51 \%)$. Les bonnes valeurs pastorales ont été obtenues dans la forêt sacrée (en moyenne $25,79 \%)$ par rapport à la jachère $(5,33 \%)$. En ce qui concerne les faibles valeurs pastorales, la moyenne obtenue dans la forêt $(13,41 \%)$ est plus faible que celle de la jachère $(18,17 \%)$. L'analyse du Tableau 4 montre que les catégories «bonne valeur pastorale » de la dépression valent 5,86 fois celle de jachère et 1,53 fois celle du glacis. Du point de vue statistique, les forêts sacrées ont présenté de meilleures catégories de bonne valeur pastorale que la jachère. Cependant, en ce qui concerne la catégorie «faible valeur pastorale », la jachère a présenté les plus fortes valeurs comparativement à la dépression et au glacis.

\section{Phytomasse}

L'évaluation de la phytomasse montre que la forêt sacrée a permis un développement significatif $(\mathrm{P}<0,05)$ des herbacées (Figure 2$)$ comparativement à celle de la jachère. Les quantités moyennes de fourrage brutes produites dans les sites sacrés sont de 4695,23 $\mathrm{kg} \pm 578$ de Matière Sèche (MS) / ha /an contre $2195,24 \pm 294 \mathrm{~kg}$ de MS / ha /an récoltées dans la jachère. Dans la zone de glacis, la phytomasse brute a été évaluée à 3364,29 $\pm 532 \mathrm{~kg}$ de MS / ha /an et $4695,23 \pm 703 \mathrm{~kg}$ de MS / ha pour la dépression.

\section{Le fourrage qualifié}

La capacité de charge pendant la saison des pluies a été de 2,50 UBT (Unité Bétail Tropical) /ha/ pendant les 4 mois de saison des pluies dans la dépression contre 1,17 UBT/ha/saison des pluies pour la jachère. 
Tableau 1 : Les principales familles d'herbacées sur les différentes unités de production fourragère (parcours).

\begin{tabular}{|c|c|c|c|c|}
\hline \multirow[b]{3}{*}{ Familles } & \multirow{2}{*}{\multicolumn{4}{|c|}{ Nombre d'espèces }} \\
\hline & & & & \\
\hline & Dépression & & & \\
\hline Poaceae & & 8 & 26 & 7 \\
\hline Fabaceae & & 2 & 8 & 4 \\
\hline Malvaceae & & 7 & 7 & 3 \\
\hline Lamiaceae & & 5 & 4 & 2 \\
\hline Amaranthaceae & & 4 & 4 & \\
\hline Rubiaceae & & 4 & 4 & 3 \\
\hline Acanthaceae & & 3 & 3 & \\
\hline Asteraceae & & 3 & 3 & 1 \\
\hline Euphorbiaceae & & 3 & 3 & \\
\hline Tiliaceae & & 3 & 2 & 2 \\
\hline Asteraceae & & 2 & 2 & \\
\hline Cochlospermaceae & & 2 & 2 & 1 \\
\hline Commelinaceae & & 2 & 1 & \\
\hline Convolvulaceae & & 2 & 2 & 1 \\
\hline Cyperaceae & & 2 & & \\
\hline Dioscoreaceae & & 2 & 1 & \\
\hline
\end{tabular}

Tableau 2 : Contribution spécifique (\%) des espèces par familles de fourrage.

\section{Forêts sacrées}

\begin{tabular}{lrrrrr}
\hline Familles & \multicolumn{1}{c}{ Dépression } & \multicolumn{1}{c}{ Glacis } & Moyenne forêts sacrées & \multicolumn{1}{c}{ Jachère } \\
\hline Poaceae & 71,70 & 60,62 & 66,16 & 33,04 \\
Légumineuses & 12,08 & 7,98 & 10,03 & 16,29 \\
Autres familles & 16,22 & 31,04 & 23,81 & 50,66 \\
\hline Total & 100,00 & 100,00 & 100,00 & 100,00 \\
\hline
\end{tabular}

Tableau 3 : Contribution spécifique (en \%) des principales espèces dans les trois parcours.

\begin{tabular}{|c|c|c|c|c|c|}
\hline \multicolumn{2}{|l|}{ Dépression } & \multicolumn{2}{|l|}{ Glacis } & \multicolumn{2}{|l|}{ Jachère } \\
\hline Espèces & Csi & Espèces & Csi & Espèces & Csi \\
\hline Zornia glochidiata*** & 18,8 & Andropogon pseudapricus* & 16,21 & Hyptis suaveolens ${ }^{\text {na }}$ & 28,15 \\
\hline Andropogon pseudapricus* & 14,15 & Zornia glochidiata*** & 12,8 & $\begin{array}{l}\text { Abutilon panosum }{ }^{\text {na }} \\
\text { Andropogon }\end{array}$ & 17,9 \\
\hline Eragrostis tenella*** & 11,5 & Eragrostis tenella*** & 11,5 & pseudapricus* & 17,14 \\
\hline Andropogon gayanus*** & 10,5 & Rottboellia exaltata $* * *$ & 9,2 & $\begin{array}{l}\text { Achyranthes aspera }{ }^{\text {a }} \\
\text { Acroceras }\end{array}$ & 10,4 \\
\hline Alysicarpus ovalifolius $* * *$ & 9,8 & $\begin{array}{c}\text { Pennisetum pedicellatum } * * * \\
104\end{array}$ & 9,1 & amplectens $^{\text {na }}$ & 6,4 \\
\hline
\end{tabular}




\begin{tabular}{|c|c|c|c|c|c|}
\hline Rottboellia exaltata*** & 9,2 & Loudetia togoensis* & 7,8 & Microchloa indica* & 3,9 \\
\hline Pennisetum polystachion $* *$ & 7,2 & Alysicarpus ovalifolius $* * *$ & 7,8 & Setaria pumila* & 2,3 \\
\hline Pennisetum & & & & Pennisetum & \\
\hline pedicellatum $* * *$ & 6,3 & Andropogon gayanus $* * *$ & 4 & pedicellatum $* * *$ & 1,4 \\
\hline Loudetia togoensis* & 3,6 & Setaria pallide-fusca * & 2,8 & & \\
\hline Setaria pallide-fusca* & 3,5 & Pennisetum polystachion $* * *$ & 2,2 & & \\
\hline Total & 89,6 & Total & 81,9 & Total & 78,95 \\
\hline
\end{tabular}

Tableau 4 : Valeur pastorale brute $(\mathrm{VPb}$ en $\%$.) des espèces par unités de production fourragère.

\begin{tabular}{lrrrrr} 
& \multicolumn{2}{l}{ Forêts sacrées } & & \\
\cline { 2 - 5 } Valeur pastorale & \multicolumn{2}{l}{ Dépression } & \multicolumn{1}{l}{ Glacis } & Moyenne F Sacrée & \multicolumn{1}{c}{ Jachère } \\
\hline Bonne $\mathrm{VPb}$ & $31,25^{\mathrm{a}}$ & $20,32^{\mathrm{a}}$ & $25,79^{\mathrm{a}}$ & $5,33^{\mathrm{c}}$ \\
Moyenne $\mathrm{VPb}$ & $11,16^{\mathrm{b}}$ & $18,38^{\mathrm{b}}$ & $14,77^{\mathrm{b}}$ & $11,01^{\mathrm{b}}$ \\
Faible $\mathrm{VPb}$ & $11,62^{\mathrm{b}}$ & $15,20^{\mathrm{b}}$ & $13,41^{\mathrm{b}}$ & $18,17^{\mathrm{a}}$ \\
Sans $\mathrm{VPb}$ & 0,00 & 0,00 & 0,00 & 0,00 \\
\hline & 54,03 & 47,90 & 50,97 & 34,51
\end{tabular}

Les valeurs portant la même lettre par colonne ne sont pas significativement différentes au seuil de 5\%. $\mathrm{VPb}$ : Valeur Pastorale brute

Tableau 5 : Capacité de charge en UBT/ha en octobre suivant les parcours.

\begin{tabular}{lrrr}
\hline Paramètres & Dépression & \multicolumn{1}{c}{ Glacis } & \multicolumn{1}{c}{ Jachère } \\
\hline Biomasse (Kg/ha) & 4695,23 & 3364,30 & 2195,25 \\
Valeur pastorale brute \% & 54,03 & 47,90 & 34,51 \\
Recouvrement \% & 98,85 & 65,42 & 58,26 \\
Valeur pastorale nette \% & 53,41 & 31,34 & 20,11 \\
Biomasse nette (Kg/ha & 2507,87 & 1054,26 & 441,36 \\
CC nette (UBT/ha/saison des pluies) & 2,50 & 1,79 & 1,17 \\
CC nette (UBT/ha/an) & 0,82 & 0,59 & 0,38 \\
\hline
\end{tabular}

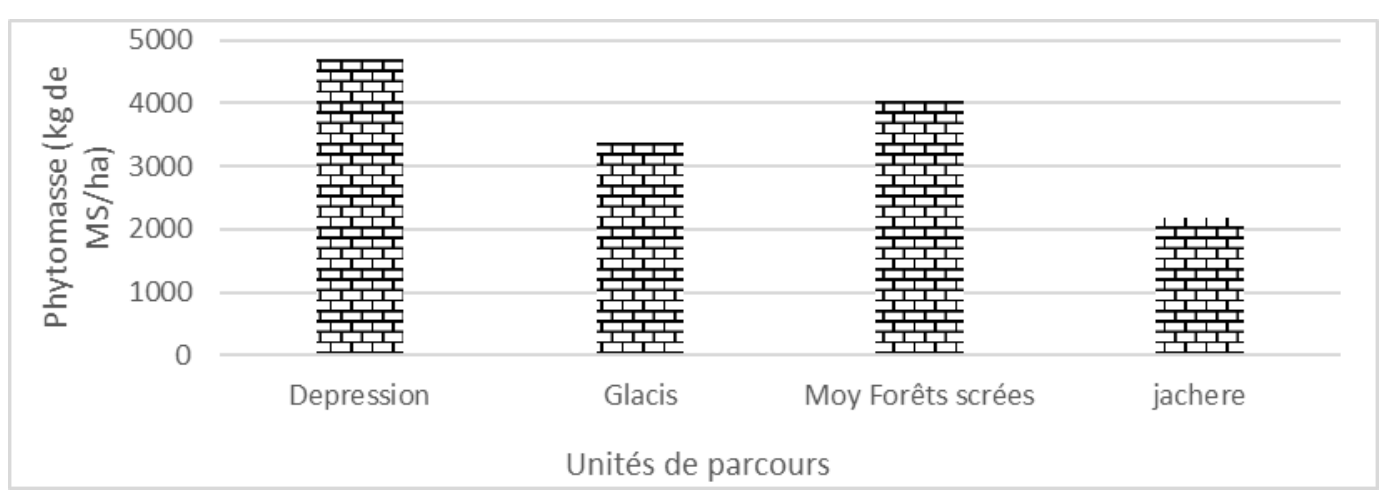

Figure 2 : Production de phytomasse brute par type d'unité de production fourragère. 


\section{DISCUSSION}

L'analyse de la composition floristique a permis d'identifier 101 espèces herbacées différentes sur l'ensemble des relevés effectués répartis dans 38 familles. Ces résultats peuvent être comparés à ceux de Yaméogo et al. (2013) qui ont travaillé dans un village de la même région sur les pâturages communautaires où 28 familles ont été inventoriées comportant 101 espèces.

L'étude qualitative de la végétation herbacée des forêts sacrées de Koupéla montre un pâturage naturel de graminées, dominé par des annuelles, avec cependant quelques pérennes (Andropogon gayanus, Andropogon ascinodis, Cymbopogon giganteus et Cymbopogon schoenanthus). La présence de Andropogon gayanus est un atout parce que selon Achard \& Banoin (2000), cette espèce est une des meilleures herbacées fourragères parmi les graminées vivaces spontanées en raison de sa haute productivité, de son excellente adaptation à la longue saison sèche, de sa vaste aire de répartition écologique et de la valeur nutritive de ses repousses. La présence de cette espèce indique une anthropisation modérée par mise en culture et pâturage (Fournier et al., 2000) ou une reconstitution d'une jachère (KaboréZoungrana et al., 1994). A. gayanus est l'espèce dominante dans la zone dépression parce qu'elle a été longtemps protégée par les us et coutumes. Quand on passe de la dépression à la zone de glacis, on observe une réduction de la contribution spécifique de l'espèce Andropogon gayanus. L'espèce n'existe pas dans la jachère parce que l'une des caractéristiques principales, soulignées par Fournier et al. (2000), est que cette Andropogoneae disparait face à une perturbation plus forte, telle une très longue culture, une jachère très courte ou un pâturage très intense.

Parmi les espèces recensées, certaines ont un indice de qualité spécifique (Is) nulle. Parmi ces espèces dont l'Is est nulle, figurent Hyptis spicigera. de la famille des Lamiaceae dans la jachère et Abutilon panosum de la famille des Malvaceae dans la zone de glacis de la forêts sacrée. La première est une espèce à tendance prolifique et envahissante. $H$. spicigera colonise actuellement les jachères de Koupéla. Non appété par les animaux, elle a une production de de biomasse fraîche estimée à $19460 \mathrm{~kg}$ par ha (Thombiano, 2008). C'est un agent de perturbation nuisible à la biodiversité autochtone des écosystèmes naturels, et, la présence de cette espèce semble, selon l'auteur cité, comme un signe général de la perturbation et de changement du milieu. Dans la zone de glacis, l'espèce Abutilon panosum est la troisième espèce du point vue de la contribution spécifique de $(13,72 \%)$. Elle est certes non appétée par les animaux mais elle a une faible biomasse par rapport à H. specigera.

Du point de vue de la valeur pastorale brute, les meilleurs résultats ont été obtenus sur la zone de dépression avec $54,03 \%$ suivi de $47,90 \%$ pour le glacis contre $34,51 \%$ pour la jachère. La partie dépression des forêts sacrées, parce que située dans un bas-fond, a plus bénéficiée des réserves hydriques qui y sont significativement plus élevées que dans les autres sites (Diouf et al., 2012). Alors les herbacées qui poussent dans ces sites profitent d'une meilleure réserve hydrique que celles des glacis. C'est pourquoi cette partie de la forêt sacrée comporte plus d'herbacées pérennes. Cette sous-unité géomorphologique bénéficie aussi de plus d'humidité, reçoit le dépôt de matières organiques et les effets de ruissellement y sont importantes (Samandoulgou et al., 2012).

En général dans les études de pâturages, les meilleurs parcours d'herbacés à bonne valeur pastorales sont les légumineuses et les Poaceae. Selon César (2005), les pâturages nord soudaniens sont d'excellentes qualités si leur valeur pastorale atteint et dépasse les $65 \%$. Dans ces conditions, seul le parcours de la zone de dépression a une valeur pastorale qui peut être qualifiée d'excellente contrairement au glacis de qualité moyenne et la jachère de faible qualité. Ce dernier parcours est dépourvu d'herbacées pérennes, ce qui est un signe de dégradation (Yameogo, 1997). 
Partant des estimations des superficies de Compaore et al. (2018) les productions de phytomasses des unités de végétation totalisent une disponibilité réelle en fourrage pour $124 \mathrm{UBT}$. Ces résultats sont meilleurs à ceux de Samandoulgou et al. (2012) dans la région des zones récupérées du Sahel située au Nord du Burkina Faso. Ce fourrage constitue un apport substantiel pour les animaux du village. Malheureusement, les pâturages naturels des forêts sacrées de Koupéla sont devenus, depuis ces dernières années communautaires et ouverts aux cheptels des terroirs voisins et des transhumants. Face à une telle pression le risque de surpâturage pourrait à terme contribuer à une dégradation irréversible des ressources fourragères des forêts sacrées surtout de la zone de glacis qui en général est le plus sensible à ce phénomène (Fabien et al., 2006 ; Ali et al., 2014).

\section{Conclusion}

La présente étude avait pour objectif d'évaluer la productivité des herbacées fourragères des forêts sacrées de Koupéla, en vue d'une élaboration future d'un système d'exploitation durable des ressources naturelles disponibles. Les résultats font ressortir la présence d'espèces pérennes appétées comme Andropogon gaynanus dans les zones de dépression et de glacis, rare dans le milieu environnant. La productivité fourragère de ces espaces sacrés est nettement supérieure à celle des jachères. Pour une production durable du fourrage sur ces sites, il faut en fonction des saisons mettre en place un cahier de charges qui règlementerait l'utilisation de ces parcours par les animaux. En perspective, il serait souhaitable qu'une étude ultérieure puisse évaluer la productivité fourragère de la strate ligneuse du terroir et estimer la contribution des résidus de culture afin de pouvoir proposer des actions de gestion globale des ressources pastorales du village. En ce qui concerne les forêts sacrées, et face à l'affaiblissement du pouvoir des gardiens de ces forêts, à la rapide croissance démographique et à l'insécurité foncière grandissante, il serait souhaitable d'envisager avec l'aide des services techniques, des ONGs et toutes les forces vives du village la création d'un comité alternatif qui aiderait à la gestion de ces forêts en collaboration avec les responsables coutumiers.

\section{CONFLIT D'INTERETS}

Les auteurs déclarent n'avoir aucun conflit d'intérêts.

\section{CONTRIBUTIONS DES AUTEURS}

La conception et le montage des protocoles ont été faits par YS, HC, SJZ et YCZ-K. L'exécution des expérimentations a été faite par YS, HC et SJZ. L'analyse des données a été faite par YCZ-K. Rédaction a été faite par YS, HC, SJZ et YCZ-K.

\section{REMERCIEMENTS}

Les auteurs remercient vivement le projet Soils of Island Forests in Africa (SOFIIA) pour l'appui technique et logistique apporté aux équipes lors des enquêtes. Ils remercient également les producteurs agricoles de Koupéla pour l'accueil chaleureux réservé aux équipes d'enquête et leur participation active à l'enquête.

\section{REFERENCES}

Achard E, Banoin M. 2000. Production Fourragère des Jachères et Transferts de Fertilité par le Bétail au Niger. In Floret \& Pontanier. Ed., $2000: 1: 546-$ 554.

Akpo LE, Masse D, Grouzis M. 2002. Durée de jachère et valeur pastorale de la végétation herbacée en zone soudanienne au Sénégal. Revue Elev. Méd. Vét. Pays Tropicaux, 55(4): 275 - 283.

Ali RKFM, Odjoubere J, Tente ABH, Sinsin AB. 2014. Caractérisation floristique et analyse des formes de pression sur les forêts sacrées ou communautaires de la Basse Vallée de l'Ouémé au Sud-Est du Bénin. Afrique Science, 10(2) : 243- 257.

Boudet G. 1991. Manuel sur les Pâturages Tropicaux et les Cultures Fourragères (2ème édn). Ministère de la Coopération et du Développement : IEMVT, Collection Manuels et Précis d'Elevage : France. 
Kaboré-Zoungrana C, Zoungrana I, Sawadogo E.1994. Variations saisonnières de la production de matière sèche et de la composition chimique d'Andropogon gayanus au Burkina Faso. Fourrages, 37: 61-74.

CAPES/RGCB. 2006. Etats des lieux des savoirs locaux au Burkina Faso. Edition CAPES/RGCB, Ouagadougou, Burkina Faso, $379 \mathrm{p}$.

Compaore H, Samandoulgou Y, Yameogo J, Zoundi JS, Kaboré-Zoungrana CY. 2018. Empreinte de l'utilisation pastorale sur les ligneux des forêts sacrées : cas de Nzoa à Koupéla dans le Centre Ouest du Burkina Faso. Série Sciences Naturelles et Appliquées - Science et Technique, Sciences Naturelles et Agronomie, 4(Spécial hors-série $n^{\circ} 4$ ).

Coulibaly A. 2016. Dynamique de la végétation ligneuse des ilots forestiers de Koupela, département de Sabou, région du Centre-Ouest, Burkina Faso. Mémoire de contrôleur des eaux et forêts, ENEF, Burkina Faso, 57 p.

Daget P, Poissonet J. 1971. Une méthode d'analyse phytosociologie des prairies: Critères. Ann. Agron., 22(1): 5-41.

Diouf M, Diouf I, Logbo J, Frederic D, Akpo LE. 2013. Déterminisme de la feuillaison de Acacia tortilis (Forsk.) dans la réserve sylvopastorale de Sogobé, Ferlo, NordSénégal ». Int. J. Biol. Chem. Sci., 7(5): 2106-2116.

DOI: http://dx.doi.org/10.4314/ijbcs.v7i5.27

Koutchika ERI, Agbani PO, Sinsin B. 2013. Influence des perturbations anthropiques sur la biodiversité des bois sacrés du Centre Bénin. Int. J. Biol. Chem. Sci., 7(1): $\quad 306-318 . \quad$ DOI : http://dx.doi.org/10.4314/ijbcs.v7i1.26

Esoh E. 2003. Plaider pour une protection des bois sacrés en Afrique noire, Actes du xIIe Congrès Forestier Mondial Québec Canada « La forêt, source de vie» Tome $\mathrm{A}$ « des forêts pour les gens». FAO, $381 \mathrm{p}$.

Esoh E. 2006. La prise en compte du magicoreligieux dans les problématiques de développement durable : le cas du
Ngondo chez les peuples Sawa du Cameroun. VertigO - la revue electronique en sciences de l'environnement, 7(3).

Fabien A, Maman Waziri M, De Boissieu D, Franck G. 2006. «Dégradation des ressources végétales au contact des activités humaines et perspectives de conservation dans le massif de l'Aïr (Sahara, Niger). VertigO - la revue electronique en sciences de l'environnement, 7(2).

Fontes J, Guinko S. 1995. Carte de la végétation et l'occupation du sol du Burkina Faso. Projet Campus, Ministère de la coopération française, $66 \mathrm{p}$.

Fournier A, Yoni M, Zombre P. 2000. Les jachères à Andropogon gayanus en savane soudanienne dans l'ouest du Burkina Faso: flore, structure, déterminants et fonction dans l'écosystème. Etudes flor. Vég. Burkina Faso, 5: 3-32.

Kabirou D. 2016. Contribution socioéconomique des relictes forestières dans le département de Sabou : village de Koupéla (Burkina Faso). Licence en économie agricole de l'environnement, Université de Koudougou, Burkina Faso, $61 \mathrm{p}$.

Kokou K, Kossi A, Klaus H. 2005. Les forêts sacrées de l'aire Ouatchi au sud-est du Togo et les contraintes actuelles des modes de gestion locale des ressources forestières, VertigO - la revue électronique en sciences de l'environnement, 6(3).

Kokou K, Sopkon N. 2006. Les forêts sacrées du couloir du Dahomey. Bois et forêts des Tropiques, (288) :15-23.

Lasseur M. 2010. Islam et christianisme en mouvement. Espace populations sociétés. DOI : 10.4000/eps.4079

Levang P, Grouzis M. 1980. Méthodes d'étude de la Biomasse herbacée de formations sahéliennes : application à la Mare d'Oursi, Haute-Volta. Acta oecological oecol. Plant., 15(3) : 231244. 
MED. 2007. Recensement général de la population et de l'habitation (RGPH) de 2006 du Burkina Faso - Résultats définitifs. Ministère de l'Economie et du Développement (MED) du Burkina Faso, $51 \mathrm{p}$.

Ouedraogo I, M. Tigabu P, Savadogo H, Compaore PCO, Ouadba JM. 2010. Land cover change and its relation with population dynamics in Burkina Faso. West Africai. Land Degrad. Develop, 21: 453-462. DOI:10.1002/ldr.981

Samandoulgou Y. 2014. Amélioration des ressources fourragères face aux risques liés au changement climatique dans la Région du Sahel Burkinabé. Thèse Doctorat Unique de l'Université Polytechnique de Bobo-Dioulasso, Burkina Faso, 164 p.

Samandoulgou Y, Kaboré-Zoungrana C, Zoundi SJ, Verbree D, Ouédraogo E, Sohoro A. 2012. Etude des effets des modes d'exploitation des pâturages aménagés en cordons pierreux associés aux sous-solages sur la production fourragère en zone sahélienne au Burkina Faso. Etudes et recherches sahéliennes Sciences Agronomiques, 18 : 09-20.

Samandoulgou Y, Kiema A, Zoundi SJ, Kabore-Zoungrana CY, Tamboura HH. 2012. Amélioration du disponible fourrager en région sahélienne par le travail du sol et l'ensemencement de Panicum laetum. Etudes et recherches sahéliennes, Sciences agronomiques, 18 : 63-72.

Thiombiaono DNE. 2008. Etude de trois espèces à tendance prolifique et envahissante dans la province du Boulkiemdé : Cassia occidentalis L.
(Caesalpiniaceae), Cassia obtusifolia L. (Caesalpiniaceae) et Hyptis suaveclens L. Poil. (Lamiaceae) ». Mémoire d'ingénieur ou développement rural option eaux et forêts, Institut du Développement Rural, Institut polytechnique de Bobo-Dioulasso, 122p.

Toutain B, Piot J. 1980. Mise en défens et possibilité de régénération des ressources fourragères sahéliennes. Etudes expérimentales dans le bassin versant de la Mare d'Oursi. Haute-Volta Institut d'élevage et de médecine vétérinaire tropicale (IEMVT), $156 \mathrm{p}$.

Toutain B, De Wispelaere G. 1978. Étude et cartographie des pâturages de l'ORD du Sahel et de la zone de délestage au NordEst de Fada N'Gourma. Haute-Volta. Institut d'élevage et de médecine vétérinaire tropicale (IEMVT) MaisonAlfort. T I. Les pâturages naturels et leur mise en valeur, $134 \mathrm{p}$.

Yameogo G, Kiema A, Yelemou B, et Ouedraogo L. 2013. Caractéristiques des ressources fourragères herbacées des pâturages naturels du terroir de Vipalogo (Burkina Faso). Int. J. Biol. Chem. Sci. 7(5): 2078-2091. DOI : http://dx.doi.org/10.4314/ijbcs.v7i5.25

Yameogo G. 1997. Etude diagnostique de la flore, de la végétation et du sol de jachères d'âges différents, dans le terroir de Thiougou (Burkina Faso). DEA, Université de Cocody, Côte d'Ivoire, 84p. 